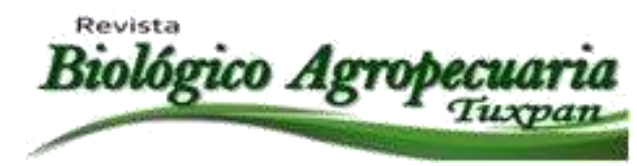

\title{
Producción de materia seca de frijol en cinco fechas de siembra durante el ciclo otoño-invierno
}

\section{en el norte de Veracruz}

Dry matter bean production in five planting dates during the autumm-winter cycle in northern Veracruz

\author{
Rodríguez Rodríguez José Raúl ${ }^{\circledR}$, Oscar H. Tosquy-Valle ${ }^{2}$ y Ernesto López-Salinas ${ }^{2}$ \\ ${ }^{1}$ Campo Experimental Ixtacuaco. INIFAP. Km. 4.5 Carretera Fed. Martínez de la Torre-Tlapacoyan, \\ Veracruz, México. A. P. 162. ${ }^{2}$ Campo Experimental Cotaxtla. INIFAP. \\ ${ }^{凶}$ Autor para correspondencia: rodriguez.jose@inifap.gob.mx
}

Recibido: 16/01/2014

Aceptado: 17/07/2014

\section{RESUMEN}

Las plantas transforman la energía luminosa en energía química aprovechable; una parte la utilizan en la respiración y el resto queda como materia seca. En el caso del frijol, la movilización de asimilados hacia el grano es muy intensa en los últimos días del ciclo del cultivo; esta parte concentra la porción más significativa del peso seco de la planta. En otoño-invierno 2012-2013, en el Campo Experimental Ixtacuaco del INIFAP, se estableció un ensayo para cuantificar la producción de materia seca total, rendimiento de grano, tasa de crecimiento diario de la biomasa e índice de cosecha en la variedad de frijol Negro Comapa con diferentes fechas de siembra las cuales fueron: $1^{\mathrm{a}}$ (20 de septiembre), $2^{\mathrm{a}}$ (27 de septiembre), $3^{\mathrm{a}}$ (4 de octubre), $4^{\mathrm{a}}$ (11 de octubre) y $5^{\mathrm{a}}$ (18 de octubre). Se utilizó un diseño experimental en bloques al azar con cuatro repeticiones; la unidad experimental fueron cuatro surcos de $5 \mathrm{~m}$ de longitud, separados a $0,60 \mathrm{~m}$, como parcela útil se tomaron los dos surcos centrales. Las variables medidas fueron: rendimiento de grano $\left(\mathrm{g} \mathrm{m}^{-2}\right)$, materia seca total $\left(\mathrm{g} \mathrm{m}^{-2}\right)$; tasa de crecimiento diario de biomasa $\left(\mathrm{g} \mathrm{m}^{-2}\right.$ día $\left.{ }^{-1}\right)$ e índice de cosecha (porcentaje de dividir el rendimiento entre la materia seca total). Se realizó análisis de varianza, las medias se compararon con la prueba de DMS $(0,05)$. La segunda y tercera fechas tuvieron la mayor acumulación de materia seca y rendimiento de grano, $\left(\right.$ ambas $\mathrm{m}^{-2}$ ), tasa de crecimiento diario de la biomasa y aceptable índice de cosecha.

Palabras clave: Phaseolus vulgaris, índice de cosecha, rendimiento biológico y de grano 


\begin{abstract}
Plants convert light energy into usable chemical energy; a part is used in breathing and rest is dry matter. In the case of beans mobilization assimilates to grain is very intense in the last days of crop cycle; this part concentrates the most significant portion of dry weight plant. In the autumm-winter 2012-2013 cycle, in the Campo Experimental Ixtacuaco del INIFAP, a trial was established to quantify dry matter production, grain yield, daily biomass growth rate and harvest index in Comapa black bean variety with different planting dates: $1^{\mathrm{a}}$ (september 20), $2^{\mathrm{a}}$ (september 27), $3^{\mathrm{a}}$ (october 4 ), $4^{\mathrm{a}}$ (october 11) and $5^{a}$ (october 18). Experimental design was a randomized blocks with four replications; the experimental unit were four rows of $5 \mathrm{~m}$ long, 0,60 apart, useful plot the two central rows. The variables measured were: grain yield $\left(\mathrm{g} \mathrm{m}^{2}\right)$, dry matter $\left(\mathrm{g} \mathrm{m}^{2}\right)$, and growth rate biomass $\left(\mathrm{g} \mathrm{m}^{2}\right.$ day $\left.^{-1}\right)$ and harvest index (percentage divided grain yield between dry matter). Analysis of variance was perfomed, comparison of means was made with DMS test $(0,05)$. The second and third dates had highest dry matter accumulation and grain yield (both $\mathrm{m}^{-2}$ ), daily growth rate biomass and a high harvest index.
\end{abstract}

Keywords: Phaseolus vulgaris, harvest index, biological and grain yield

\section{INTRODUCCION}

La agricultura es considerada la ciencia de la "cosecha" de energía luminosa, la cual se transforma en energía química aprovechable; una parte es utilizada para la respiración y el resto queda como materia seca, la cual se distribuye en los diversos órganos de las plantas (Kohashi, 1990; Richards, 2000). En el caso del frijol, la movilización de fotoasimilados hacia el grano es muy intensa durante los últimos días del ciclo del cultivo; en esta parte se concentra la porción más significativa del peso seco de la planta (White, 1991; Coelho y Benedito, 2008). Una medida de eficiencia de la distribución de materia seca en el cultivo de frijol es el índice de cosecha, el cual es la relación del rendimiento entre el peso seco total, y señala el porcentaje de la biomasa que el cultivo dedica a la producción de grano en relación con las demás estructuras de la planta (Escalante y Kohashi, 1993; Araújo y Teixeira, 2012). Cuando un cultivo tiene un adecuado suministro de agua y nutrimentos, se maximiza el proceso de fotosíntesis, respiración y transpiración, los cuales son precursores de la formación de materia seca Verdoot et al. (2004), esto crea una buena adaptación y desarrollo del cultivo, y provoca una relación positiva entre el rendimiento, la tasa de crecimiento de la biomasa, y su acumulación total (Scully et al. 1991); Amanullah y Asim, (2011). El objetivo del presente trabajo, fue cuantificar la producción de materia seca, rendimiento de grano, índice de cosecha y tasa de crecimiento diario de la biomasa en la variedad de frijol Negro Comapa en cinco fechas de siembra.

\section{MATERIALES Y METODOS}

El experimento se estableció en terrenos del Campo Experimental Ixtacuaco del INIFAP, ubicado a $20^{\circ} 09^{\prime}$ latitud norte y $97^{\circ}$ 04' longitud oeste, a una altitud de $92 \mathrm{~m}$. Durante el ciclo otoño invierno 2012-2013 se

Revista Científica Biológico Agropecuaria Tuxpan 2 (1)

ISSN: 2007-6940 
sembró la variedad Negro Comapa, de grano negro, opaco y pequeño, en cinco fechas de siembra: $1^{\mathrm{a}}$ (20 de septiembre), $2^{\mathrm{a}}$ (27 de septiembre), $3^{\mathrm{a}}$ (4 de octubre), $4^{\mathrm{a}}$ (11 de octubre) y $5^{\mathrm{a}}$ (18 de octubre). Se utilizó el diseño experimental de bloques al azar con cuatro repeticiones; se surcó a 0,60 m entre hileras; la unidad experimental fueron cuatro surcos de $5 \mathrm{~m}$ de longitud, y como parcela útil se utilizaron los dos surcos centrales; los parámetros de respuesta fueron: rendimiento de grano $\left(\mathrm{g} \mathrm{m}^{-2}\right)$ (muestra de 10 plantas con competencia completa por parcela), estas mismas plantas se colocaron en una estufa a 80 ${ }^{0} \mathrm{C}$ durante 72 horas, para deshidratarlas y determinar así su producción de materia seca total $\left(\mathrm{g} \mathrm{m}^{-2}\right)$; índice de cosecha (porcentaje de dividir rendimiento de grano entre la materia seca total); tasa de crecimiento de biomasa ( $\mathrm{g}$ $\mathrm{m}^{-2}$ día $^{-1}$ ), que resulta de dividir la materia seca total entre el número de días a madurez. Como variables explicativas se registraron la precipitación pluvial $(\mathrm{mm})$ y la temperatura $\left({ }^{\mathrm{O}} \mathrm{C}\right)$. Con el paquete la UANL, Versión 2,5, se realizó análisis de varianza de las variables de respuesta y las medias se compararon con la prueba de Diferencia Mínima Significativa $(0,05)$.

\section{RESULTADOS Y DISCUSION}

En el Cuadro 1, se muestra que hubo
efecto significativo para todas las
características evaluadas en las diferentes
fechas de siembra. La producción de materia
seca total por metro cuadrado en la segunda y
tercera fechas ( 27 de septiembre y 4 de octubre,
respectivamente) fueron superiores a las demás;
según señalan Ruíz et al. (1999) que para un
buen desarrollo del cultivo del frijol se
requieren 50 a 90 mm de precipitación pluvial
durante la etapa reproductiva y temperaturas
medias mensuales entre 20 y $28{ }^{\circ} \mathrm{C}$, y en las
fechas señaladas anteriormente se registraron

precipitaciones y temperaturas desde floración hasta llenado de vainas de $82,3 \mathrm{~mm}$ y $23,0{ }^{\circ} \mathrm{C}$ en la segunda fecha, y $60,6 \mathrm{~mm}$ y $22,8^{\circ} \mathrm{C}$ en la tercera, es decir, ambas fechas tuvieron condiciones favorables para una alta producción de biomasa Dapaah et al. (2000); Padilla et al. (2008); debido a ello tuvieron un buen suministro de fotosintatos; esto propicia que durante este período el frijol tenga una mayor acumulación de biomasa, Chavarín et al. (2008); sobre este aspecto menciona Kohashi (1990), que durante esta etapa existe un mayor consumo de asimilados, especialmente por las vainas y los granos, debido a esta alta demanda, se incrementa la tasa fotosintética en las hojas que satisfacen dicha exigencia, y se pueden producir más nutrimentos. La segunda y tercera fechas obtuvieron estadísticamente también mayor rendimiento de grano por metro cuadrado, lo cual estuvo favorecido por la mayor cantidad de materia seca total que acumularon (White 1991; Dapaah et al. 2000; Barrios-Gómez et al. 2010). En el mismo Cuadro, se observa que existió muy poca diferencia entre los índices de cosecha de las diferentes fechas, y coincide con lo encontrado por Araújo y Teixeira, (2012); Amanullah y Asim (2011), en el sentido de que este es un carácter en el que no existe mucha variabilidad en frijol; la segunda fecha de siembra tuvo estadísticamente más eficiencia en canalizar carbohidratos hacia el grano, por su alta acumulación de materia seca en la semilla, respecto a la materia seca total, lo cual es consistente con lo encontrado por Grafton y Schneiter (1985); Coelho y Benedito (2008) y Amanullah y Asim (2011); sin embargo fue similar estadísticamente a la primera y tercera; los índices de cosecha entre las diversas fechas oscilaron entre 0,64 y 0,70 lo cual indica que todas tuvieron una aceptable formación de vainas y semillas en relación con el desarrollo vegetativo del cultivo (White, 1991).

\section{Revista Científica Biológico Agropecuaria Tuxpan 2 (1) ISSN: 2007-6940}


Cuadro 1. Materia seca total, rendimento de grano, índice de cosecha y tasa de crecimiento diario de la biomasa de frijol variedad Negro Comapa en cinco fechas de siembra en el norte de Veracruz. Campo Experimental Ixtacuaco-INIFAP. Ciclo otoño-invierno 2012-2013.

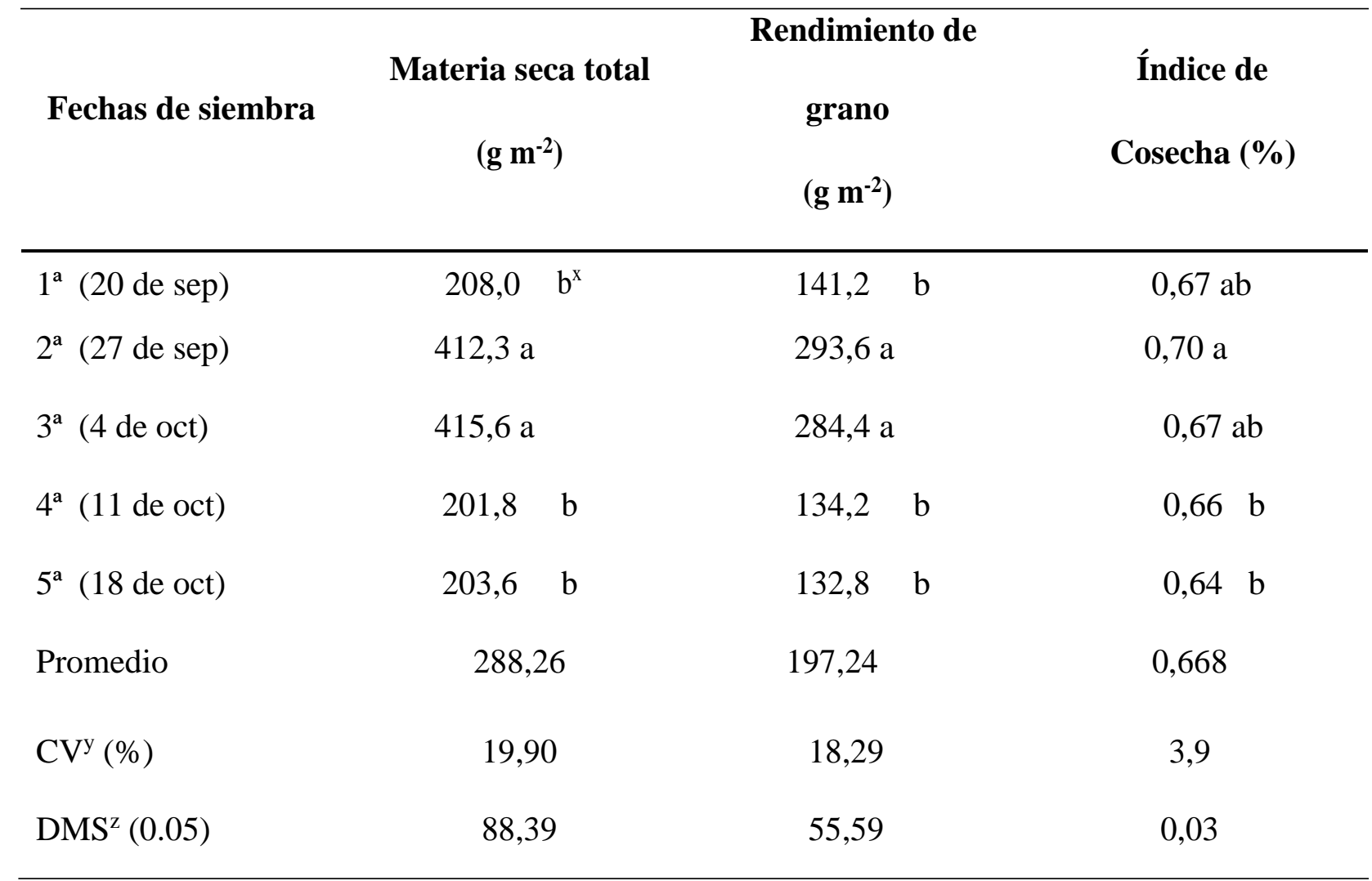

${ }^{\mathrm{x}}$ Medias con la misma letra dentro de columnas, son estadísticamente iguales; $\mathrm{CV}^{\mathrm{y}}=$ Coeficiente de variación; $\mathrm{DMS}^{\mathrm{Z}}=$ Diferencia Mínima Significativa.

En la Figura 1 se observa la tasa de crecimiento diario de la biomasa; la segunda y tercera fechas de siembra tuvieron significativamente el mayor incremento en gramos de materia seca en cada día, esto es indicativo de que las condiciones durante el crecimiento y desarrollo de las plantas interaccionaron bien con el ambiente, el cual les proporcionó capacidad y eficiencia para convertir la radiación fotosintéticamente activa en energía aprovechable y alto rendimiento de grano, lo cual está respaldado por lo que señalaron sobre este aspecto Scully y Wallace (1990); Dapaah et al. (2000); Verdoot et al.

(2004). La tasa de crecimiento del cultivo, la producción de biomasa total y la producción de grano, tuvieron una respuesta semejante entre ellos, ya que según mencionan Scully et al (1991); Amanullah y Asim (2011), existe una fuerte relación entre estas características, de ahí su tendencia a tener igual comportamiento en las fechas de siembra mencionadas (27 de septiembre y 4 de octubre, respectivamente).

\section{Revista Científica Biológico Agropecuaria Tuxpan 2 (1)} ISSN: 2007-6940 


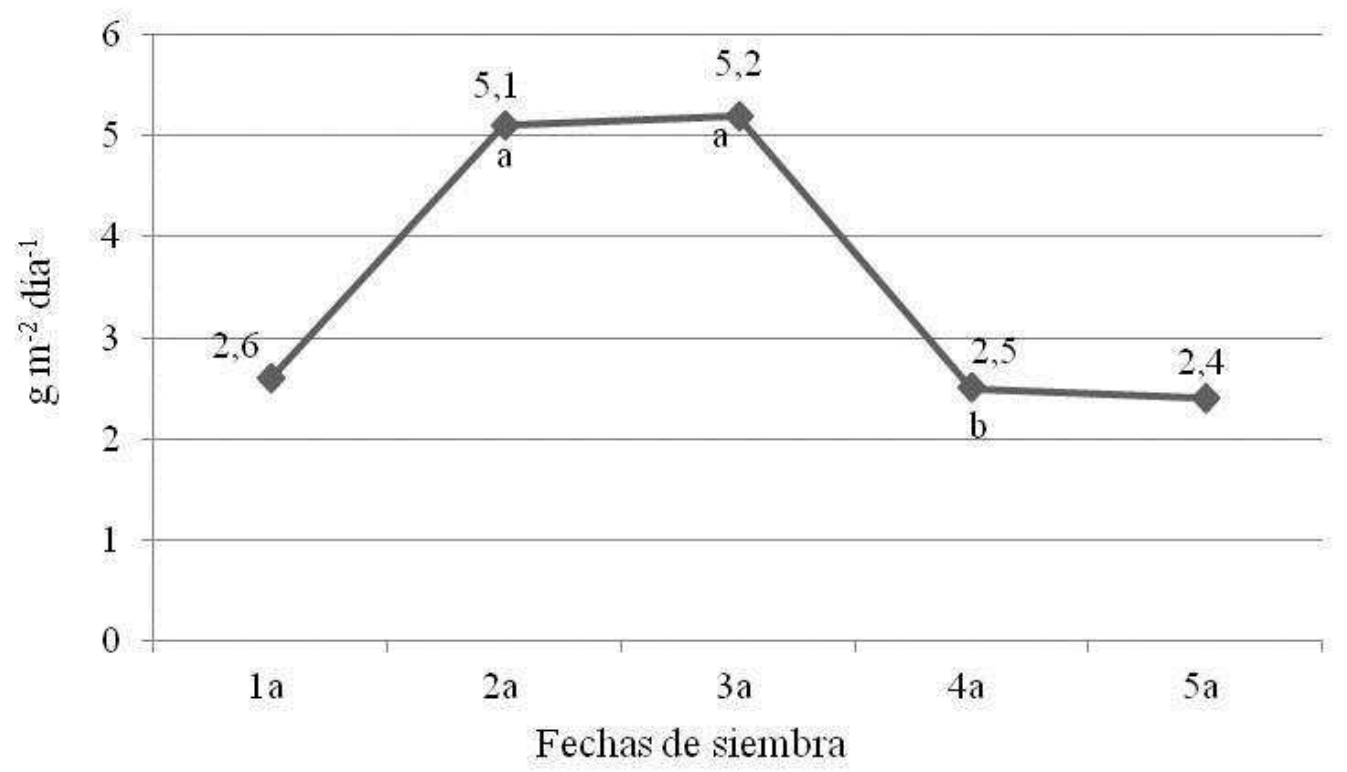

Figura 1. Tasa de crecimiento de la biomasa de frijol en cinco fechas de siembra.

\section{CONCLUSIONES}

La segunda y tercera fechas (27 de septiembre y 4 de octubre, respectivamente) obtuvieron la mayor acumulación de materia seca total y rendimiento ambas por metro cuadrado, la más alta tasa de crecimiento diario de la biomasa y aceptable porcentaje de índice de cosecha.

\section{LITERATURA CITADA}

Amanullah, M. y Asim, M. 2011. Evaluation of common bean germplasm collected from the Neglected Pockets of Northwest Pakistan at Kalam. Pak. J. Bot. 43(1): 213-219.

Araújo, A. y Teixeira, M. 2012. Variabilidade dos índices de colheita de nutrientes em genótipos de feijoeiro e sua relação com a produção de grãos. Revista Brasileira de Ciência do Solo. 36(1): 137-146. https://doi.org/10.1590/s0100-06832012000100015
Barrios-Gómez, E., López, C., Kohashi, J., Acosta, J., Miranda, S. y Mayek, N. 2010. Rendimiento de semilla, y sus componentes en frijol Flor de Mayo en el centro de México. Agrociencia. 44: 481-489. https://doi.org/10.15517/rac.v35i1.6690

Chavarín, I., Lépiz, R. y López, J. J. 2008. Fenología y acumulación de materia seca en variedades de frijol arbustivo de diferente hábito de crecimiento. XIX Semana Nacional de la Investigación Científica. In: Avances en la Investigación científica en el Centro Universitario de Ciencias Biológicas y Agropecuarias (CUCBA). Universidad de Guadalajara. Guadalajara, México. p. 25-30.

https://doi.org/10.32870/e-cucba.v0i7.63

Coelho, C. y Benedito, V. 2008. Seed development and reserve compound accumulation in common bean (Phaseolus vulgaris L.). Seed Science and Biotechnology. 2(2): 42-52. 
Dapaah, H., Mckenzie, B. y Hill, G. 2000. Infuence of sowing date and irrigation on the growth and yield of pinto beans (Phaseolus vulgaris) in a subhumid temperate environment. Journal of Agricultural Science. Cambridge University Press. 134:33-43. https://doi.org/10.1017/s0021859699007248

Escalante, J. A. y Kohashi, J. 1993. El rendimiento y crecimiento del frijol. Manual para la toma de datos. Colegio de Postgraduados. Montecillo, Texcoco, México. $84 \mathrm{p}$. https://doi.org/10.24850/j-tyca-2020-01-03

Grafton K. y Schneiter, A. A. 1985. Effect of planting dates on yield and other agronomics traits of dry bean. Farm Research. 42 (6):11-13.

Kohashi, J. 1990. Aspectos de la Morfología y Fisiología del frijol (Phaseolus vulgaris L.) y su rendimiento. Centro de Botánica. Colegio de Postgraduados. Montecillo, Texcoco, México. 44 p. https://doi.org/10.24850/j-tyca-2018-05-07

Padilla, J., Osuna, E. y Acosta, E. 2008. Effect of sowing date on seed yield of early and late dry bean cultivars at highlands of Mexico. Bean Improvement Cooperative. 51:230-231.

Richards, R. 2000. Selectable traits to increase crop photosynthesis and yield for grain crops. Journal for Experimental Botany. 51:447-458.

https://doi.org/10.1093/jexbot/51.suppl_1.447

Ruiz C., Medina, G. J. A., González, A. G., Ortiz, T. I. J., Flores L. C., Martínez, P. H. E. y Bierly, M. K. F. 1999. Requerimientos Agroecológicos de Cultivos. Instituto Nacional de Investigaciones Forestales, Agrícolas y Pecuarias. Centro de Investigación Regional del Pacífico Centro, Campo
Experimental Centro de Jalisco. Guadalajara, Jalisco, México. 324 p. https://doi.org/10.20937/rica.2019.35.01.18

Scully, B. y Wallace, D. 1990. Variation in and relationship of biomass, growth rate, harvest índex, and phenology to yield of common bean. J. Amer. Soc. Hort. Sci. 115(2): 218-225.

Scully, B., Wallace, D. y Viands, D. 1991. Heritability and correlation of biomass, growth rates, harvest index, and phenology to the yield of common beans. J. Amer. Soc. Hort. Sci. 116(1):127-130. https://doi.org/10.21273/jashs.115.2.218

Verdoodt, A., van Ranst, E. y Ye, L. 2004. Daily simulation of potential dry matter production of annual field crops in Tropical Environments. Agronomy Journal. 96 (6): 1739-1753. https://doi.org/10.2134/agronj2004.1739

White, J. 1991. Conceptos básicos en fisiología de frijol. In: López, M., F. Fernández y A. van Schoonhoven (eds). Frijol: Investigación y Producción. Centro Internacional de Agricultura Tropical (CIAT). Cali, Colombia. p. 43-60. https://doi.org/10.17138/tgft(2)130-132 
Copyright (c) 2014 José Raú1 Rodriguez Rodriguez, Oscar Hugo Tosquy Valle y Emesto López Salinas

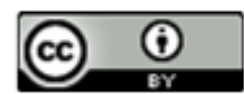

Este texto está protegido por una licencia Creative Common 4.0.

Usted es libre para Compartir —copiar y redistribuir el material en cualquier medio o formato- y Ad aptar el documento —remezclar, transformar y crear a partir del material一 para cualquier propósito, inchso para fines comerciales, siempre que cumpla la condición de:

Atribución Usted debe dar crédito a la obra original de manera adecu ada, proporcionar un enlace a la licencia, e indicar si se han realizado cambios. Puede hacerlo en cualquier forma razonable, pero no de forma tal que sugiera que tiene el apoyo del licenciante o lo recibe por el usoque hace de la obra.

Resumendelicencia - Textocompletodelalicencia 